\title{
Nachruf auf Erik Olin Wright (1947-2019)
}

\author{
Hans-Peter Müller
}

Online publiziert: 16. April 2020

(C) Der/die Autor(en) 2020

Wenn man Erik Olin Wright und sein Werk in nur zwei Begriffen zu charakterisieren hätte, so hießen sie „Klasse“ und „Utopie“. Über sein soziologisches Lieblingsthema „Klasse“ hat er insgesamt acht Bücher verfasst, über „Utopie“ eines, das gleichsam sein politisches Vermächtnis darstellt. Das Buch Reale Utopien dürfte in Zukunft die Handreichung sein, wenn man lernen will, wie eine fundamentale Gesellschaftsveränderung schrittweise und ohne ideologische Selbstverblendung ins Werk zu setzen ist.

Am 9. Februar 1947 in Berkeley, Kalifornien, in eine bildungsbürgerliche jüdische Familie geboren, machte Wright seinen B.A. in „Social Studies“ 1968 am Harvard College, dann 1970 einen zweiten B.A. in „History“ am Balliol College im britischen Oxford, um schließlich sein Studium 1976 mit einem Ph.D. in Berkeley abzuschließen. Bereits im gleichen Jahr trat er eine Professur an der University of Madison, Wisconsin, an, der er Zeit seines Lebens, mit Ausnahme einer Gastprofessur in Berkeley 1987/88, verbunden bleiben sollte. Da hatte er seine politische Sozialisation schon hinter sich. Wie man seinem autobiografischen Essay „Falling into Marxism; choosing to stay" für Alan Sicas und Stephen Turners Band The Disobedient Generation: Social Theorists in the Sixties (2005) entnehmen kann, engagierte er sich schon früh in der sozialen Bürgerrechtsbewegung und hörte 1963 in Washington Martin Luther Kings berühmte Rede „I have a dream“. Um die Einberufung zum Militärdienst hinauszuzögern, schrieb er sich 1968 nach Abschluss des Harvard College in ein unitarisches theologisches Seminar der „Starr King School for the Ministry“ in Berkeley ein, bekam dann aber ein Stipendium für die Oxford University, wo er sich u.a. mit der englischen puritanischen Revolution beschäftigte. In Oxford war es Steven Lukes, der den jungen Wright zu einer verstärkten

H.-P. Müller (四)

Humboldt-Universität zu Berlin, Berlin, Deutschland

E-Mail: hpmueller@sowi.hu-berlin.de 
Auseinandersetzung mit den Ideen von Marx anregte. Im Herbst 1970 kehrte er in die USA und an die unitarische Bildungsanstalt zurück, und als 1971 die Regeln zur Einberufung zum Militär auf ein Lotterie-Verfahren umgestellt wurden und er eine günstige Nummer zog, verließ er das theologische Seminar und wechselte als ,graduate student“ zur Soziologie. Wie die 68er-Generation allgemein, wird auch ihn der Vietnam-Krieg vollends politisch mobilisieren. Neben seiner soziologischen Doktorarbeit setzte er sich mit Politischer Ökonomie auseinander, er trat in das Redaktionskollektiv der Zeitschrift Kapitalistate ein und beteiligte sich an der Gründung der „Union of Marxist Social Scientists“.

Auf sein erstes Buch The Politics of Punishment aus dem Jahr 1973 über das amerikanische Gefängniswesen ließ er 1978 Class, Crisis and the State folgen. Dieser Band machte ihn rasch auch international bekannt. Wright hatte sein erstes Lebensthema gefunden: das Problem der Klassen und der Klassenstrukturanalyse. Er schließt sich als eins der jüngsten Mitglieder einer Gruppe von analytischen Marxisten an, die einen ,no-bullshit“-Marxismus zum Programm erheben und ab 1979 jährlich Konferenzen abhalten. Das Marx'sche Forschungsprogramm wird ernstgenommen, aber mit den Mitteln der analytischen Philosophie gründlich auseinandergenommen, um die Spreu vom Weizen zu trennen und dem Marxismus zu mehr wissenschaftlicher Präzision, Klarheit und Strenge zu verhelfen. $\mathrm{Zu}$ dieser Gruppe von marxistischen Intellektuellen gehörten u. a. Gerald A. Cohen (Karl Marx's Theory of History, 1978), John Roemer (A General Theory of Exploitation and Class, 1982) und Jon Elster (Making Sense of Marx, 1985), um nur die bekanntesten Protagonisten und ihre wichtigsten Bücher zu nennen. Innerhalb der Gruppe wird es Wrights Aufgabe, das Thema „Klasse“ zu traktieren. Das tat er nicht nur analytisch, sondern mithilfe quantitativer Methoden, die seine Thesen empirisch untermauern sollten. Sein Hauptaugenmerk galt der Mittelklasse, der Achillesferse der Marx'schen Klassentheorie, die dichotomisch angelegt ist. Seine Auflösung des Rätsels lautet: Es gibt in kapitalistischen Gesellschaften zwangsläufig widersprüchliche Klassenpositionen im System der Klassenbeziehungen; die Mittelklasse ist zum Teil kapitalistisch, zum Teil proletarisch. Beharrlichkeit, Sorgfalt, analytische Raffinesse und empirische Validierungsversuche zeichnen seine Klassenanalysen aus, die in weiteren sieben Büchern seinen Niederschlag fanden: Class Structure and Income Determination (1985), Classes (1985), The Debate on Classes (zusammen mit anderen Autoren, 1990), Interrogating Inequality (1994), Class Counts (1997), Approaches to Class Analysis (Hrsg., 2005) und Understanding Class (2015). Während er in den 1970er-Jahren noch viele Mitstreiter hatte, weil „Klasse“ damals ein angesagtes Thema war, setzte mit dem Niedergang des Marxismus und schließlich dem Zusammenbruch des sozialistischen Weltsystems ein wachsendes Desinteresse an der Problematik ein. Wrights analytische Obsession fußte indes auf der Überzeugung, dass der Kapitalismus ein unhintergehbares Klassenverhältnis mit Ausbeutung begründet, das wirkliche Gleichheit und Demokratie unmöglich macht.

Wenn das so ist, dann gilt es, nach Alternativen Ausschau zu halten. Das führte Wright zu seinem zweiten Lebensthema: den Utopien und ihren Realisierungschancen. Während seine Klassenanalysen dem sozialstrukturellen Rückgrat der modernen Gesellschaft nachgehen, diskutiert er unter dem Stichwort „Utopia“ die sozialen und kulturellen Auswege aus dem Kapitalismus und die Strategien seiner Transformati- 
on in den ,Sozialismus“ als Verkörperung der ,guten Gesellschaft“. Im Jahre 1991 begründete er das „Real Utopias Project“. Die Zeiten hatten sich freilich gegenüber den 1960er- und 1970er-Jahren gründlich geändert. Die alte Ordnung der drei Welten - die erste Welt des Westens mit Kapitalismus und Demokratie, die zweite Welt des real existierenden Sozialismus mit einem autokratischen Einparteiensystem und die dritte Welt der Entwicklungsländer - war 1989 zusammengebrochen. Es schien nur noch die eine Welt von Kapitalismus, Demokratie und Individualismus zu existieren, zu dem der Rest der Welt nur noch aufzuschließen brauchte. Dieser „Monovision" einer alternativlosen Welt des globalen Neoliberalismus hielt Wright nach dem Motto des Weltsozialforums „Another world is possible“ alternative Entwürfe entgegen, die er „Realutopien“ nannte. Insgesamt sieben Sammelbände wurden im Rahmen des „Real Utopias Project“ veröffentlicht. ${ }^{1}$ Sein eigenes Buch Envisioning Real Utopias von 2010, auf Deutsch unter dem Titel Reale Utopien. Wege aus dem Kapitalismus 2017 erschienen, markiert sicherlich den Höhepunkt dieses Projekts.

Als Wright im Mai 2017 auf Einladung der Rosa-Luxemburg-Stiftung vor großem Publikum an der Humboldt-Universität zu Berlin seinen Vortrag ,Reale Utopien und die Überwindung des Kapitalismus“ hielt, konnte man erleben, warum alle, die ihn kannten, ihn als freundlich, neugierig, zugänglich und einfühlsam beschrieben haben. Nachdem ich Person und Werk vorgestellt hatte, legte er los - und wie! Fast eine Stunde hielt er sein Publikum im Bann, und was zunächst wie ein abstraktes, durch und durch trockenes akademisches Sujet klingen mochte, erstand vor der Zuhörerschaft zu einer mit viel Optimismus und Sympathie vorgetragenen Alternative zu den jetzigen gesellschaftlichen Verhältnissen. Wright plädierte für eine graduelle, nicht disruptive Transformation, nicht für eine Revolution im klassischen Sinn, sondern für eine friedliche Transition. Wenn erst einmal die Wünschbarkeit, Machbarkeit und Erreichbarkeit eines konkreten transformatorischen Projekts hinreichend realitätsnah festgestellt sind, kann man beherzt die Nischen des Kapitalismus besetzen. Je mehr Erfolge sich einstellen, umso mehr Nachahmer wird es geben und sich sozialistische Ideen und Praktiken weiter ausbreiten. Freilich: Wrights pluraler, demokratischer Sozialismus hat nichts mit dem real existierenden Vorläufer des Partei- und Staatssozialismus zu tun, den Wright kurzerhand „Etatismus“ nennt und als Sackgasse verwirft. Ihm ging es um ein „socialist empowerment“ von unten, eine sukzessive Unterordnung der ökonomischen und der staatlichen Macht unter die gesellschaftliche Macht. Wer das Studium des umfangreichen Bandes Reale Utopien scheut, sich jedoch mit dem Grundgedanken seines Transformationskonzeptes vertraut machen möchte, dem sei seine „Presidential Address“ als Präsident der „American Sociological Association“ aus dem Jahre 2011 zur Lektüre empfohlen, die auch auf Youtube zu hören und zu sehen ist (Wright 2013).

Fünfzehn Bücher und Hunderte von Artikeln hat Wright in seinem reichen akademischen Leben verfasst. Sein sechzehntes Buch How to be an Anticapitalist in the Twenty-First Century konnte er noch kurz vor seinem Tod fertigstellen; es liegt bereits auch auf Deutsch vor (Wright 2019). In ihm propagiert er einen radikaldemokratischen Marktsozialismus. Es ist das geistige Vermächtnis eines der letzten großen marxistischen Intellektuellen der Vereinigten Staaten. Wright dürfte häufig

\footnotetext{
1 Siehe https://www.versobooks.com/series_collections/2-the-real-utopias-project.
} 
belächelt worden sein für sein obstinates Festhalten am Marxismus, und nur sein freundliches Wesen hat ihn wohl vor der Exkommunikation aus dem Kreis der professionellen Soziologie bewahrt. Er selbst hat die Absetzbewegung innerhalb der Kollegenschaft in die „Professionalität“ kritisch beobachtet, als die radikalen politischen Zeiten, in denen der Marxismus opportun war, abgeklungen waren. Aufgrund seiner wissenschaftlichen und politischen Redlichkeit ist er dem Prinzip des „Dennoch" gefolgt, hat es sich mit dieser Positionierung also bewusst schwerer gemacht. „Making one's life more difficult in this way, however, is not a sign of masochism; it is a strategy that makes it harder to slide inadvertently into a theoretical and intellectual practice that is overwhelmed by its professional acceptability. The pressures for mild, nonconfrontational, acceptable scholarship are enormous, and situating one's work firmly in a radical oppositional current is one way of partially neutralizing those pressures“" (Wright 2005, S. 336).

Am 23. Januar 2019 ist er seiner Leukämie-Krankheit erlegen. Wir werden Erik Olin Wright weiter lesen, sowohl als einen der größten Klassentheoretiker des 20. Jahrhunderts als auch als Streiter für die Verwirklichung realer Utopien im 21. Jahrhundert.

Funding Open Access funding provided by Projekt DEAL.

Open Access Dieser Artikel wird unter der Creative Commons Namensnennung 4.0 International Lizenz veröffentlicht, welche die Nutzung, Vervielfältigung, Bearbeitung, Verbreitung und Wiedergabe in jeglichem Medium und Format erlaubt, sofern Sie den/die ursprünglichen Autor(en) und die Quelle ordnungsgemäß nennen, einen Link zur Creative Commons Lizenz beifügen und angeben, ob Änderungen vorgenommen wurden.

Die in diesem Artikel enthaltenen Bilder und sonstiges Drittmaterial unterliegen ebenfalls der genannten Creative Commons Lizenz, sofern sich aus der Abbildungslegende nichts anderes ergibt. Sofern das betreffende Material nicht unter der genannten Creative Commons Lizenz steht und die betreffende Handlung nicht nach gesetzlichen Vorschriften erlaubt ist, ist für die oben aufgeführten Weiterverwendungen des Materials die Einwilligung des jeweiligen Rechteinhabers einzuholen.

Weitere Details zur Lizenz entnehmen Sie bitte der Lizenzinformation auf http://creativecommons.org/ licenses/by/4.0/deed.de.

\section{Literatur}

Wright, E. O. (2005). Falling into Marxism; choosing to stay. In A. Sica \& S. Turner (Hrsg.), The disobedient generation. Social theorists in the sixties (S. 325-349). Chicago: University of Chicago Press.

Wright, E. O. (2013). Transforming capitalism through real utopias. American Sociological Review, 78, $1-25$.

Wright, E. O. (2019). Linker Antikapitalismus im 21. Jahrhundert. Was es bedeutet, demokratischer Sozialist zu sein. Hamburg: VSA. 